\title{
Different origins of the ferromagnetic order in (Ga,Mn)As and (Ga,Mn)N
}

\author{
Małgorzata Wierzbowska* \\ Physics Department, Trinity College, Dublin 2, Ireland \\ Daniel Sánchez-Portal \\ Unidad de Fisica de Materiales, Centro Mixto CSIC-UPV/EHU and Donostia International Physics Center (DIPC), \\ Paseo Manuel de Lardizabal 4, 20018 Donostia-San Sebastián, Spain \\ Stefano Sanvito ${ }^{\dagger}$ \\ Physics Department, Trinity College, Dublin 2, Ireland
}

(Received 9 June 2004; revised manuscript received 29 September 2004; published 17 December 2004)

\begin{abstract}
The mechanism for the ferromagnetic order of $(\mathrm{Ga}, \mathrm{Mn}) \mathrm{As}$ and $(\mathrm{Ga}, \mathrm{Mn}) \mathrm{N}$ is extensively studied over a vast range of Mn concentrations. We calculate the electronic structures of these materials using density functional theory in both the local spin density approximation (LSDA) and the local density approximation (LDA) $+U$ scheme, that we have now implemented in the code SIESTA. For $(\mathrm{Ga}, \mathrm{Mn}) \mathrm{As}$, the LDA $+U$ approach leads to a hole mediated picture of the ferromagnetism, with an exchange constant $N \beta=-2.8 \mathrm{eV}$. This is smaller than that obtained with LSDA, which overestimates the exchange coupling between Mn ions and the As $p$ holes. In contrast, the ferromagnetism in wurtzite $(\mathrm{Ga}, \mathrm{Mn}) \mathrm{N}$ is caused by the double-exchange mechanism, since a hole of strong $d$ character is found at the Fermi level in both the LSDA and the LDA $U$ approaches. In this case the coupling between the Mn ions decays rapidly with the Mn-Mn separation. This suggests a two phases picture of the ferromagnetic order in $(\mathrm{Ga}, \mathrm{Mn}) \mathrm{N}$, with a robust ferromagnetic phase at large $\mathrm{Mn}$ concentration coexisting with a diluted weak ferromagnetic phase.
\end{abstract}

DOI: 10.1103/PhysRevB.70.235209

PACS number(s): 75.30.Et, 71.15.Mb, 71.15.Ap

\section{INTRODUCTION}

In recent years there was a rapid development in the growth and characterization of diluted magnetic semiconductors (DMS). ${ }^{1}$ These materials are obtained by doping with transition metals ordinary III-V semiconductors ${ }^{2}$ and recently also transition metal oxides. ${ }^{3}$ The novel aspect of the DMS is the interplay between the electronic functionality of a semiconductor with magnetic properties. For example the possibility of tailoring the ferromagnetic Curie temperature $\left(T_{c}\right)$ by electron gating has already been demonstrated. ${ }^{4}$ It is then clear that the measurement of a spontaneous magnetization, although a good test for ferromagnetism, is not direct proof that a material is a DMS. Therefore some other measurements such as the anomalous Hall effect ${ }^{2}$ or x-ray magnetic circular dicrohism $(\mathrm{XMCD})^{5}$ must be used to demonstrate the interaction between the magnetic and the electronic degrees of freedom. To date only a few materials, including (Ga,Mn)As and (In,Mn)As, have convincingly passed this test, ${ }^{5}$ but unfortunately none of them present a $T_{c}$ above room temperature. This, of course, is a critical requisite for future devices.

New excitement came with the synthesis of ferromagnetic (Ga,Mn)N (Refs. 6-9) with $T_{c}$ well above room temperature. High $T_{c}$ in this material was somehow expected after the predictions of Dietl et al., ${ }^{10}$ who calculated the magnetic properties of various semiconductors incorporating $\mathrm{Mn}$, and concluded that wide gap semiconductors might offer better possibility for $T_{c}$ above room temperature. Dietl's calculations are based on the Zener ${ }^{11}$ model of ferromagnetism, where the localized 5/2 spins of the Mn ions are antiferromagnetically coupled with the spins of the free holes, giving rise to an effective $\mathrm{Mn}-\mathrm{Mn}$ ferromagnetic interaction. Interestingly high $T_{c}(\mathrm{Ga}, \mathrm{Mn}) \mathrm{N}$ does not show any evidence for anomalous hole effect, nor for any hysteretic XMCD signal coming from the valence $\mathrm{GaN}$ electrons. ${ }^{12}$ This seems to suggest that in the case of $(\mathrm{Ga}, \mathrm{Mn}) \mathrm{N}$ the agreement with the Dietl's theory is somehow coincidental.

Since the experimental situation is not conclusive, ab initio methods are important for understanding the main features of these novel materials, and for establishing the validity of models based on effective Hamiltonians. ${ }^{10,13}$ So far a large number of density functional theory (DFT) calculations for a wide range of DMS have been published (see Ref. 14 for a review). Almost all the calculations to date are based on the local spin density approximation (LSDA) and here we list the main results for $(\mathrm{Ga}, \mathrm{Mn}) \mathrm{As}$ and $(\mathrm{Ga}, \mathrm{Mn}) \mathrm{N}$ :

(1) $(\mathrm{Ga}, \mathrm{Mn})$ As is a half-metal with a magnetic moment of $4 \mu_{B}$ per $\mathrm{Mn}$ in the cell. ${ }^{15-18}$

(2) In $(\mathrm{Ga}, \mathrm{Mn})$ As the local magnetic moment at the $\mathrm{Mn}$ site is larger than $4 \mu_{B}$ and the Fermi level lies below the top of the majority valence band. This sustains the idea of a hole with spin antiferromagnetically coupled to that of the Mn. In addition an induced magnetic moment antiparallel to that of the $\mathrm{Mn}$ is found at the As sites neighboring the Mn ions.

(3) The hole in $(\mathrm{Ga}, \mathrm{Mn})$ As has a rather large $d$ component as the result of a considerable $p$ - $d$ interaction.

(4) For $(\mathrm{Ga}, \mathrm{Mn}) \mathrm{As}$ an estimation of the exchange constant $N \beta$ gives a value of about $-4.5 \mathrm{eV}$ (Ref. 15), which is considerably larger than that given by most experimental determinations and the value used in model Hamiltonian calculations. ${ }^{10,13}$

(5) $(\mathrm{Ga}, \mathrm{Mn}) \mathrm{N}$ is also a half-metal with a magnetization of $4 \mu_{B}$ per $\mathrm{Mn}$ in the cell. ${ }^{19}$ 
(6) The valence band of $(\mathrm{Ga}, \mathrm{Mn}) \mathrm{N}$ is not spin splitted and the Fermi level lies in a rather narrow impurity band. ${ }^{19}$

(7) The magnetic impurity band in $(\mathrm{Ga}, \mathrm{Mn}) \mathrm{N}$ has a strong $d$ character and the magnetic moment at the Mn sites is consistent with a Mn $d^{4}$ configuration. ${ }^{16}$

Very recently we have investigated whether some of these common features are pathological of the use of the LSDA. In particular, since the LSDA tends to underestimate electron localization and to overestimate the $p$ - $d$ hybridization, one may cast some doubts on its quantitative predictions. We have carried out electronic structure calculations for both $(\mathrm{Ga}, \mathrm{Mn}) \mathrm{As}$ and $(\mathrm{Ga}, \mathrm{Mn}) \mathrm{N}$ by using the self-interaction corrected LDA method (LDA-SIC). ${ }^{20,21}$ The main findings are that, on the one hand the electronic structure of $(\mathrm{Ga}, \mathrm{Mn}) \mathrm{As}$ are rather similar in LSDA and LDA-SIC, although the second predicts a much weaker $p$ - $d$ hybridization at the top of the valence band with consequent reduction of the valence band spin splitting. This, of course, means that the exchange constant $N \beta$ is smaller than that predicted by the LSDA. On the other hand, for $(\mathrm{Ga}, \mathrm{Mn}) \mathrm{N}$ LDA-SIC shows a strong orbital ordering with a convincing evidence of a Mn $d^{4}$ configuration. Although this can be reinterpreted as $\mathrm{Mn} d^{5}$ plus a localized $d$ hole, it is clear that no holes are left in the GaN valence band and an itinerant free-hole-mediated picture of ferromagnetism is not sustainable. A somehow similar picture has been found in a series of recent works using the $\mathrm{LDA}+U$ method. ${ }^{22-25}$

Unfortunately, due to its computational overheads we have not been able to investigate the details of the Mn-Mn interaction with the LDA-SIC method yet. The present paper seeks to fill this gap. We have implemented the LDA $+U$ scheme in the localized atomic orbital DFT code SIESTA, ${ }^{26,27}$ and then used its computational capability to investigate the magnetic properties of $(\mathrm{Ga}, \mathrm{Mn}) \mathrm{As}$ and $(\mathrm{Ga}, \mathrm{Mn}) \mathrm{N}$. The method, although it introduces two phenomenological parameters (the Coulomb $U$ and the exchange $J$ constants), allows us to perform large scale calculations and therefore to investigate the $\mathrm{Mn}-\mathrm{Mn}$ interaction over a broad range of $\mathrm{Mn}$ concentrations.

The paper is organized as follows. In the next section we will briefly discuss our computational details and we will justify the values used for the LDA $+U$ phenomenological parameters. Then we will present our results for both $(\mathrm{Ga}, \mathrm{Mn}) \mathrm{As}$ and $(\mathrm{Ga}, \mathrm{Mn}) \mathrm{N}$. Finally, we will conclude. Details on the implementation of the LDA $+U$ method in SIESTA are described in the Appendix.

\section{COMPUTATIONAL DETAILS}

All the calculations of this work are performed with the density functional code SIESTA. ${ }^{26,27}$ SIESTA has been specially optimized to deal with very large systems. It uses a very efficient localized atomic orbital basis set ${ }^{28-30}$ and norm conserving pseudopotentials in the separate Kleinman-Bylander form. ${ }^{31}$ It is therefore ideal to simulate arrangements of hundreds and even thousands of atoms, hence DMS with low Mn concentrations ${ }^{14}$ and related systems. ${ }^{32}$

We use conventional scalar relativistic Troulier-Martins pseudopotentials ${ }^{33}$ with nonlinear core corrections. ${ }^{34}$ The ref- erence electronic configurations for the pseudopotentials are $2 s^{2} 2 p^{3} 3 d^{0}(\mathrm{~N}), 4 s^{2} 4 p^{3} 3 d^{0}$ (As), $4 s^{2} 4 p^{0} 3 d^{5} 4 f^{0}(\mathrm{Mn})$, with s/p/d cutoff radii 1.14/1.14/1.14 a.u. $(\mathrm{N}), 1.9 / 2.18 / 2.5$ a.u. (As), and $s / p / d / f$ radii for Mn 1.98/2.18/1.88/1.88 a.u. We treat the $4 s$ and $4 p$ electrons of Ga as valence electrons and we leave the $3 d$ in the core. Therefore the pseudopotential is constructed for $4 s^{2} 4 p^{1} 3 d^{0}$ with $s / p / d$ cutoff radii 2.1/2.5/2.98 a.u. We also checked whether or not the inclusion of $3 d$ electrons in the valence changes the relevant properties. In GaAs, although this was shown to be important for the geometry optimization and the high pressure phases, ${ }^{35}$ it does not seem to be particularly relevant for the physics at the Fermi level under normal pressure conditions. In contrast, in GaN the $3 d$ states lie about $3 \mathrm{eV}$ below the N-2s states. ${ }^{36}$ LSDA sets erroneously their position within the $\mathrm{N}-2 s$ band and one may suspect that this will affect somehow the physics at the Fermi level. We fix this wrong alignment by applying $U$ corrections to the $\mathrm{Ga} 3 d$ states and find that, although now the bands have the correct position, the Fermi sphere is not modified. Therefore we decided not to include $\mathrm{Ga} 3 d$ states in the valence in order to save computational time and memory. Note that the same choice is presently adopted in most of the calculations of this type. ${ }^{19}$

As far as the lattice structure is concerned, all the calculations assume the experimental geometry for both zinc blende $\operatorname{GaAs}\left(a_{0}=5.65 \AA\right)$ and wurtzite $\mathrm{GaN}(a=3.189 \AA$, $c=5.185 \AA$, and $u=0.377)$. ${ }^{37}$ We then perform supercell simulations, where the supercells are constructed as integer multiple of either the primitive or in the case of zinc blende the cubic cell.

Turning our attention to the basis set it is worth pointing out that SIESTA uses a flexible multiple- $\zeta$ basis set $^{38}$ of numerical atomic orbitals. Our calculations use double- $\zeta$ for the $s$ and $p$ shells of any element and a triple- $\zeta$ basis set for the Mn $3 d$ shell. Details of the relevant basis cutoff radii and their optimization has been already given elsewhere. ${ }^{15}$

Although SIESTA is a very powerful and flexible package, it includes only basic features to tackle magnetic systems. In particular it can only use the LSDA or the generalized gradient approximation (GGA) for the exchange correlation potential. No schemes to deal with strong electron correlations are included. Since we believe that these may play an important role in determining the magnetic properties of III-V DMS, we decided to implement the LDA $+U$ scheme in SIESTA, using the functional proposed by Anisimov et al. ${ }^{39,40}$ Details of the implementations are discussed in the Appendix.

Since the LDA $+U$ method is essentially empirical, in the sense that the values of the Coulomb and exchange constants $U$ and $J$ must be provided, we have explored several choices of these parameters. Our fitting criterion was to choose $U$ and $J$ in order to obtain the best energy position of the Mn $d$ band compared with that of available photoemission data. ${ }^{41}$ This fit fixes $U$ and $J$, respectively, to 4.5 and $1.0 \mathrm{eV}$. In addition to the correct positioning of the Mn $d$ band we find that this set of parameters also reproduces accurately the band structure of $(\mathrm{Ga}, \mathrm{Mn})$ As that we have obtained with the LDA-SIC method. ${ }^{20}$ Finally it is worth noting that variations of $J$ of the order of $50 \%$ about $1.0 \mathrm{eV}$ do not produce significant changes and that we have assumed that the same set 
of parameters can be used also for $\mathrm{Mn}$ in $(\mathrm{Ga}, \mathrm{Mn}) \mathrm{N}$. Here we also present some results for $U=8 \mathrm{eV}$ in order to give a better explanation of the trends.

\section{RESULTS AND DISCUSSION}

A schematic Hamiltonian for describing the main interaction in III-V DMS is

$$
\mathcal{H}=\mathcal{H}_{0}+N \beta \vec{S} \cdot \vec{s}
$$

where $\mathcal{H}_{0}$ is the Hamiltonian for the host semiconductor and $N \beta$ is the exchange constant between the Mn spin $\vec{S}$ and the spin $\vec{s}$ of some type of carriers. When the relevant carriers are holes this Hamiltonian leads to two possible scenarios depending on the magnitude of $N \beta / \Delta$, where $\Delta$ is the valence band bandwidth. In the case of $N \beta \ll \Delta$ the Mn-Mn coupling is mediated by free carriers and can be treated in terms of the mean-field Zener ${ }^{11}$ model.

In contrast if $N \beta \sim \Delta$ an impurity band forms at the top of the valence band with a strong Mn $d$ character and the effective coupling is double-exchange-like. ${ }^{42}$ Finally in the case $N \beta \gg \Delta$, when the holes are strongly localized at the $\mathrm{Mn}$ sites, a magnetic polaron can form and the interaction is expected to be rather short range and described by percolation theory. ${ }^{42}$ In addition to this picture, if the Mn $d$ levels lie at midgap with weak coupling with the carriers of either the conduction or valence band of the host semiconductor, the entire use of the Hamiltonian (1) can be questioned and the ferromagnetism might be described by a Zhang-Rice polaron. ${ }^{43}$ One of the aims of this work is to distinguish between these multiple options.

\section{A. (Ga,Mn)As: Electronic structure}

In Fig. 1 we show the density of the states (DOS) for $3.125 \%(\mathrm{Ga}, \mathrm{Mn})$ As obtained with the LSDA and the LDA $+U$ schemes, respectively. Both methods result in a halfmetal with a magnetic moment of $4 \mu_{B}$ per $\mathrm{Mn}$ in the cell. The difference between LSDA and LDA $+U$ is in the position of the Mn-derived $d$ DOS and its contribution to the top of the majority spin valence band. LDA $+U$ shifts the center of the majority Mn $d$ band to lower energies and now the DOS shows a strong Mn- $d$ peak at about $4 \mathrm{eV}$ below Fermi level $\left(E_{F}\right)$. This is a feature that agrees perfectly with the photoemission spectra of Ref. 41. Moreover this downshift changes the contribution of the Mn $d$ levels to the DOS at $E_{F}$ which is substantial in the LSDA (18\% of the total DOS at $\left.E_{F}\right)$ and small in the LDA $+U(7 \%)$. These features are a consequence of the enhanced localization of the Mn $d$ shells in real space. The magnetic moment per Mn atom calculated from the Mülliken population analysis ${ }^{44}$ is $4.21 \mu_{B}$ for LSDA and $4.71 \mu_{B}$ for LDA $+U$ with $U=4.5 \mathrm{eV}$. LDA $+U$ therefore gives a clear confirmation of the Mn $d^{5}$ configuration.

In addition there is always an induced magnetic moment at the As sites, which is antiparallel to that of the Mn ion. In this case the LSDA and LDA $+U$ behaviors are somehow different. In LSDA we find a rapid decay of the induced magnetic moment with the distance between the As and the Mn ions. This is $0.068 \mu_{B}$ for the first neighbor, $0.011 \mu_{B}$ for

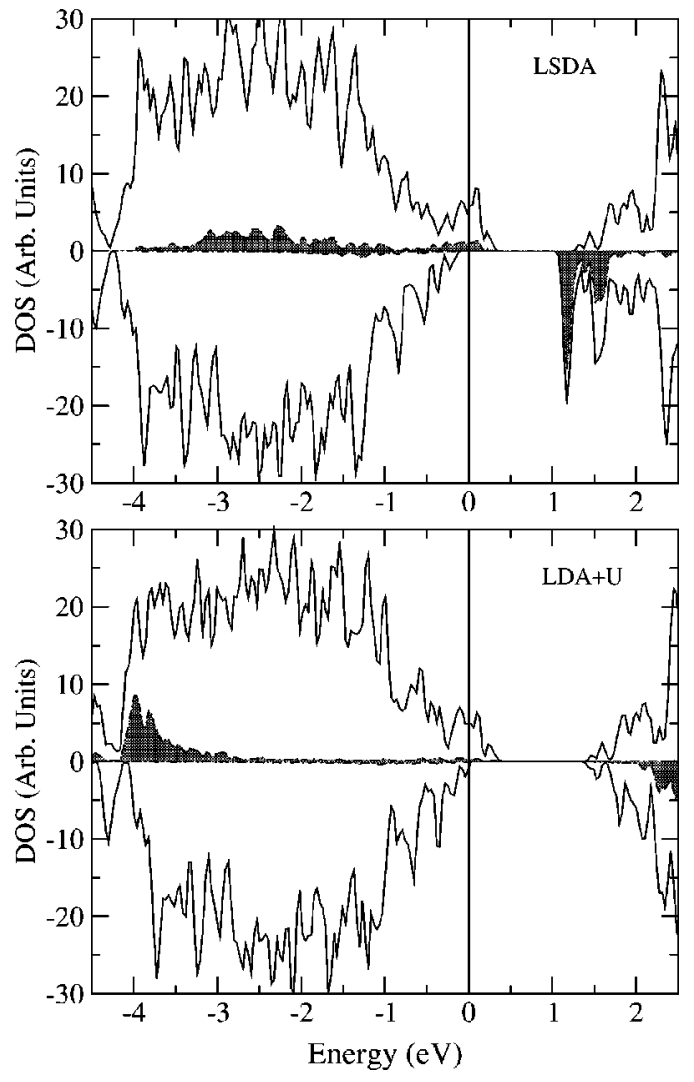

FIG. 1. DOS for $3.125 \%(\mathrm{Ga}, \mathrm{Mn})$ As calculated with both the LSDA (upper panel) and the LDA $+U$ (lower panel) schemes. The solid line represents the total DOS and the dashed area the contribution from the Mn $d$ electrons. The values of $U$ and $J$ are, respectively, 4.5 and $1 \mathrm{eV}$. The vertical line denotes the position of the Fermi level $\left(E_{F}=0 \mathrm{eV}\right)$.

the second, $0.006 \mu_{B}$ for the third. In contrast $\mathrm{LDA}+U$ gives a larger magnetic moment for the first neighbor $\left(0.106 \mu_{B}\right)$, but then this becomes almost constant with the distance from the $\mathrm{Mn}$ ion $\left(\sim 0.015 \mu_{B}\right)$.

Finally it is worth noting that the $\mathrm{LDA}+U$ pushes the unoccupied $\mathrm{Mn} d$ states in the minority band to higher energies and therefore the minority conduction band bottom changes from $d$-like to $s p$-like. Despite aforementioned features, it seems that taking into account strong Coulomb interaction in the Mn $3 d$ shell, does not change much the qualitative LSDA picture of $(\mathrm{Ga}, \mathrm{Mn})$ As near $E_{F}$. In order to appreciate the differences between the two schemes and distinguish between the different scenarios described at the beginning of this section we need to investigate in more details the magnetic interaction.

\section{B. (Ga,Mn)As: Magnetic interaction}

We first decide to evaluate the Mn- $d$ /free-hole exchange constant $N \beta$. Before starting we would like to note that $N \beta$ is not a physical observable. Therefore it cannot be measured directly but must be inferred from some other quantity. In particular the Hamiltonian (1) solved within the mean-field approximation, leads to the prediction of a linear dependence 


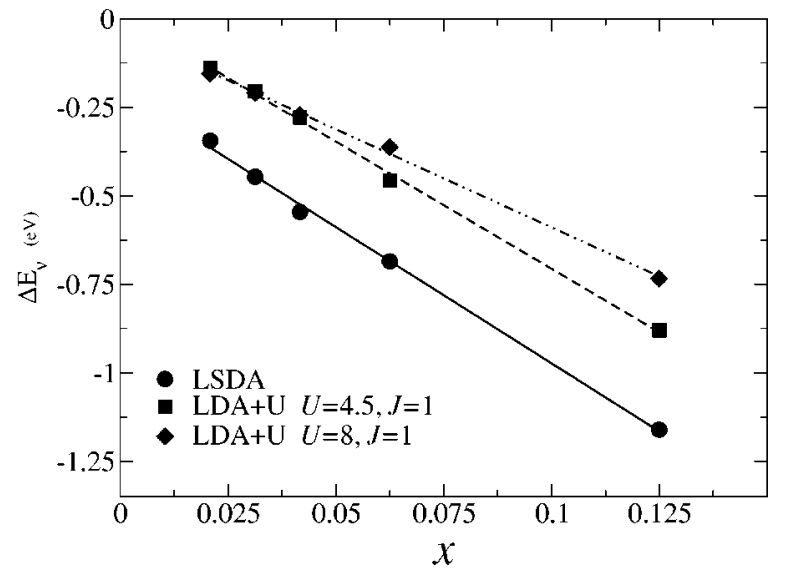

FIG. 2. Valence band spin splitting $\Delta E_{v}$ for $(\mathrm{Ga}, \mathrm{Mn}) \mathrm{As}$ at various Mn concentrations. Results for LSDA and LDA $+U$ with $U$ $=4.5 \mathrm{eV}, J=1 \mathrm{eV}$ and at $U=8 \mathrm{eV}, J=1$. The straight lines represent our best fit taken over all the concentration range.

of the spin splitting of the valence band $\Delta E_{v}$ upon the Mn concentration $x$

$$
N \beta=\frac{\Delta E_{v}}{x \cdot\langle S\rangle},
$$

where $\langle S\rangle$ is the average spin of the Mn site (5/2 assuming a Mn $d^{5}$ configuration). ${ }^{15}$ This is a quantity that can be easily calculated within our approach. Of course the idea of extracting $N \beta$ from Eq. (2) underpins the assumption that DFT and the Hamiltonian $\mathcal{H}$ solved in the mean-field approximation lead to the same physics.

In Fig. 2, we present $\Delta E_{v}$ for $x$ equal to $0.021,0.031$, $0.042,0.063$, and 0.125 obtained by substituting one $\mathrm{Ga}$ atom with $\mathrm{Mn}$ in supercells containing, respectively, 96, 64, 48,32 , and 16 atoms. It is easy to note that the slope of the linear dependence of $\Delta E_{v}$ upon $x$ decreases when going from LSDA to LDA $+U$, and furthermore it becomes smaller as $U$ gets larger. We also note that only in the case of $U=4.5 \mathrm{eV}$ the linear interpolation presents the correct limit $\Delta E_{v} \rightarrow 0$ for $x \rightarrow 0$, while this is not found for the LSDA or for other values of $U$. Although multiple scattering corrections which reproduce the appropriate behavior for $x \rightarrow 0$ can be added to our analysis, ${ }^{15,45}$ this is an interesting result in itself. In fact the value of $U=4.5 \mathrm{eV}$ also gives the correct position of the Mn $d$ states, and produces band structures that closely agree with those calculated with our parameter free LDA-SIC method. ${ }^{20}$ We therefore conclude that the LDA $+U$ method with $U=4.5 \mathrm{eV}$ and $J=1 \mathrm{eV}$ accurately describes the electronic structures of $(\mathrm{Ga}, \mathrm{Mn}) \mathrm{As}$, which in turn is also consistent with the mean-field theory. ${ }^{10}$ We then extract the value of $N \beta$ using Eq. (2). In doing this we have observed that, although it is possible to fit very accurately for either large $(x=0.031,0.042,0.063$, and 0.125$)$ or small $(x=0.021$, $0.031,0.042$, and 0.063$)$ Mn concentrations, a good fit over the whole concentration range is not satisfactory. This is somehow expected since for small concentrations multiple scattering corrections are relevant and for large ones, the Mn ions cannot be considered as a small perturbation to the
GaAs electronic structure. We therefore always calculate two values of $N \beta$ corresponding to the two different fits.

In this way we find $-3.0<N \beta<-2.8 \mathrm{eV}$ for $\mathrm{LDA}+U$ $(U=4.5 \mathrm{eV})$, which must be put in relation with the values of $-3.25<N \beta<-3.0 \mathrm{eV}$ and $-2.23<N \beta<-2 \mathrm{eV}$ extracted, respectively, from the LSDA and from $\mathrm{LDA}+U$ with $U$ $=8 \mathrm{eV}$.

We believe that our value of $N \beta \sim-2.8 \mathrm{eV}$ is the appropriate value to use in the Hamiltonian (1). Let us point out the exact meaning of our statement. We state that in a perfectly ordered, defect-free $(\mathrm{Ga}, \mathrm{Mn})$ As crystal, where every $\mathrm{Mn}$ ion occupies a Ga site and therefore donates a local spin $5 / 2$ and a hole, the spin splitting of the valence band will be that given in Fig. 2 for $U=4.5 \mathrm{eV}$. Then, if we also suppose that $\Delta E_{v}$ can be described by the Hamiltonian $\mathcal{H}$ of Eq. (1), the correct "bare" value for $N \beta$ is in the range $-3.0<N \beta$ $<-2.8 \mathrm{eV}$. In contrast if one wants to extract a value of $N \beta$ from experimental data it must be remembered that the actual value entering in the definition of the observables (for instance, the band spin splitting) is not the "bare" one, but an "effective" value that somehow takes into account effects such as disorder, presence of compensating defects and so on. These defects, if abundant, may lead to drastic modifications of the valence band top, with the resulting change in $N \beta$. Note that in this case $N \beta$ will depend not only on the concentration but also on the specific nature of the defects, their positions and their charging state. Therefore it should not be surprising that these values do not agree with the "bare" one obtained here, and that the measurements of different observables give different values of $N \beta$. Magnetotransport gives values ranging from $1.5 \mathrm{eV}$ (Ref. 46) to 3.3 $\mathrm{eV}^{47}$ exciton splitting gives $N \beta=2.5 \mathrm{eV}$ (Ref. 48) and core level photoemission $N \beta=-1.2 \mathrm{eV} .{ }^{49}$

To further investigate the effects of strong on-site correlation on the electronic structure of $(\mathrm{Ga}, \mathrm{Mn})$ As we have analyzed the effective Mn-Mn interaction. As usual we consider supercells where we now include two Mn ions per cell. ${ }^{50} \mathrm{We}$ calculate the total energy of the cell either imposing a ferromagnetic $\left(E_{\mathrm{FM}}\right)$ or antiferromagnetic $\left(E_{\mathrm{AF}}\right)$ alignment of the magnetization of the Mn ions. Then our total energy calculations are fitted to a simple Heisenberg model, in which the energy can be written as

$$
E=-\sum_{i>j} J\left(r_{i j}\right) \vec{S}_{i} \cdot \vec{S}_{j}
$$

where $J\left(r_{i j}\right)$ is the exchange constant as a function of the Mn-Mn separation $r_{i j}=\left|\vec{R}_{i}-\vec{R}_{j}\right|$ and $\vec{S}_{i}$ is the Mn spin at site $\vec{R}_{i}$. Here we assume $\vec{S}_{i}=\langle\vec{S}\rangle=5 / 2$ independently on the atomic position. This is consistent with the magnetic moment per Mn obtained from our DFT calculations. Moreover we consider $J(r)$ to be rather short ranged by setting $J(r)=0$ for $r>10.0 \AA$. Our results for $x=0.063$ for both LSDA and $\mathrm{LDA}+U$ with $U=4.5 \mathrm{eV}$ as a function of the Mn-Mn separation are presented in Fig. 3.

Both LSDA and LDA $+U$ show a decay of the magnetic coupling strength between two Mn ions as their separation increases. In addition we also observe some oscillating behavior. This is expected when dealing with a carrier- 


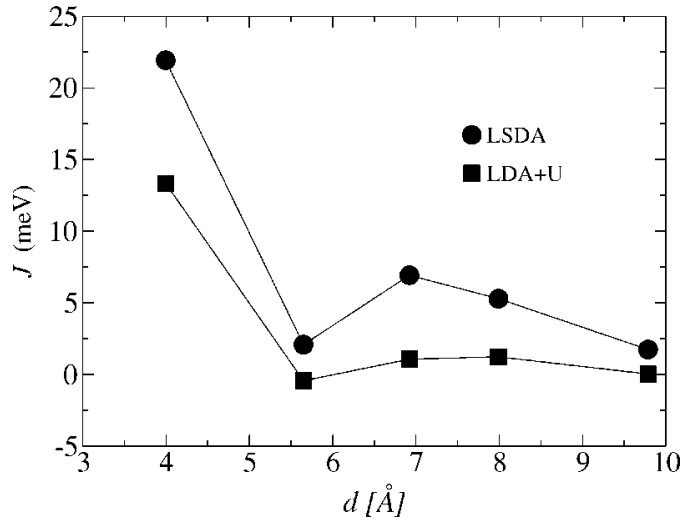

FIG. 3. Exchange parameter $J$ as a function of the Mn-Mn separation $d$ for $x=0.063$. The results are for LSDA and LDA $+U$ with $U=4.5 \mathrm{eV}$ and $J=1.0$.

mediated ferromagnetism, although our $J(r)$ cannot be fitted to an RKKY-like expression due to the lack of antiferromagnetic interaction (positive $J$ 's). It is interesting to note that our results are rather similar to those obtained previously with LSDA, ${ }^{51}$ which have been interpreted in terms of ferromagnetic paths through As sites. The main difference between the LSDA and the LDA $+U$ results is a substantial reduction of the Mn-Mn interaction when strong correlation is included.

A rough estimation of the possible $T_{c}$ can be obtained by using the mean-field approximation. ${ }^{52}$ This involves the sum of the exchange parameters over all the cation sites. From Fig. 3 it is then clear that $\mathrm{LDA}+U$ predicts a $T_{c}$ for $\sim 6 \%$ $(\mathrm{Ga}, \mathrm{Mn}) \mathrm{As}$ considerably smaller than that predicted by LSDA.

In conclusion for $(\mathrm{Ga}, \mathrm{Mn}) \mathrm{As}$ both LSDA and $\mathrm{LDA}+U$ give a picture of hole-mediated ferromagnetism. However

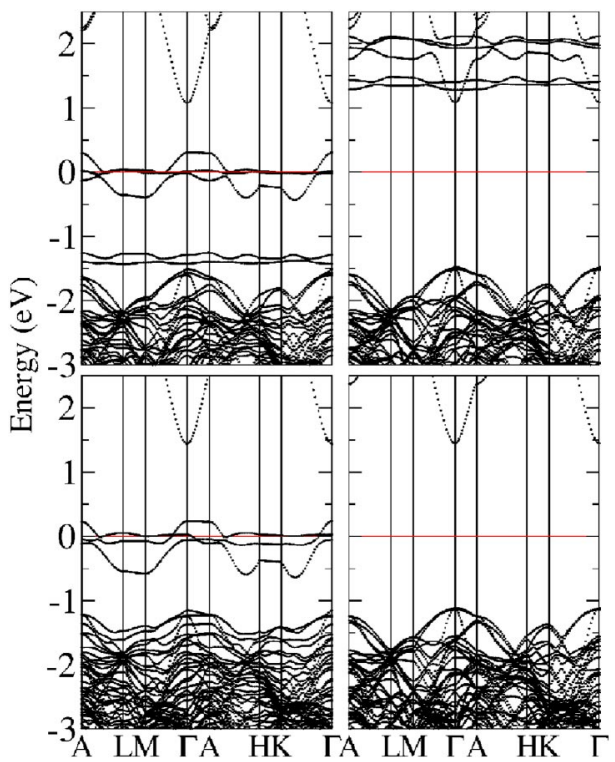

FIG. 4. Band structure of $3.125 \%(\mathrm{Ga}, \mathrm{Mn}) \mathrm{N}$ calculated with LSDA (upper panel) and LDA $+U(U=4.5 \mathrm{eV}, J=1.0 \mathrm{eV}$ ) (lower panel). The picture on the left (right) is for majority (minority) spins. The horizontal line denotes the position of the Fermi level $\left(E_{F}=0 \mathrm{eV}\right)$.
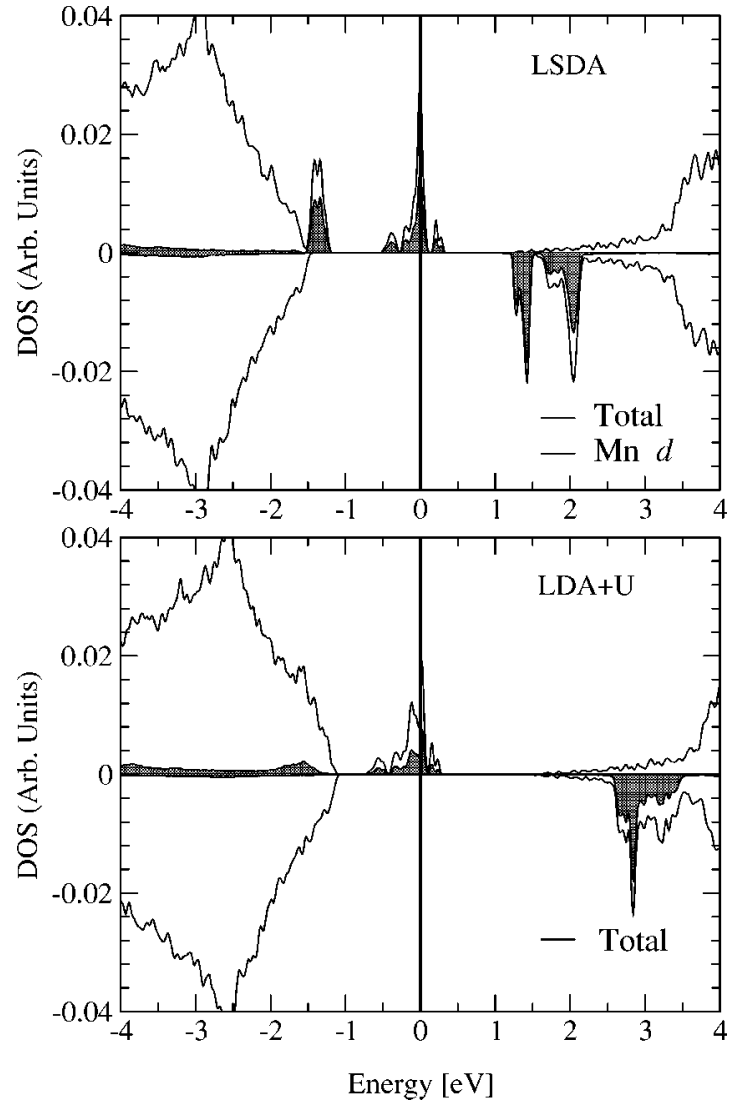

FIG. 5. DOS of $3.125 \%(\mathrm{Ga}, \mathrm{Mn}) \mathrm{N}$ calculated with LSDA (upper panel) and $\mathrm{LDA}+U(U=4.5 \mathrm{eV}, J=1.0 \mathrm{eV})$ (lower panel). The solid line represents the total DOS and the dashed area the contribution from the Mn $d$ electrons. The vertical line denotes the position of the Fermi level, that we set to $0 \mathrm{eV}$.

the inclusion of strong on-site repulsion at the Mn sites reduces the $p$ - $d$ hybridization at the top of the valence band with a consequent reduction of the effective Mn-Mn interaction.

\section{C. $(\mathrm{Ga}, \mathrm{Mn}) \mathrm{N}$ : Electronic structure}

The band structure and the corresponding DOS for $(\mathrm{Ga}, \mathrm{Mn}) \mathrm{N}$ with a $\mathrm{Mn}$ concentration of $3.125 \%$ (1 Mn ion in a 64 atom GaN cell) are presented respectively in Figs. 4 and 5. For both LSDA and LDA $+U$ and in contrast with zinc blende $(\mathrm{Ga}, \mathrm{Mn}) \mathrm{As}$, in the wurtzite $(\mathrm{Ga}, \mathrm{Mn}) \mathrm{N}$ the $\mathrm{Mn}$ $d$-derived states appear in the middle of the $\mathrm{GaN}$ gap in the majority spin band. The Fermi level cuts through a triplet with mainly $\mathrm{Mn} d$ and $\mathrm{N} p$ character and no free holes are left in the valence band, which in turn does not spin split.

From the pictures it is clear that also $(\mathrm{Ga}, \mathrm{Mn}) \mathrm{N}$ is a half metal with a total magnetization of the unit cell of $4 \mu_{B}$, as in the $(\mathrm{Ga}, \mathrm{Mn}) \mathrm{As}$ case. However, for $(\mathrm{Ga}, \mathrm{Mn}) \mathrm{N}$ the Mülliken population gives us a magnetic moment per $\mathrm{Mn}$ ion of $3.74 \mu_{B}$ and $3.86 \mu_{B}$, respectively, for LSDA and LDA $+U$. This is consistent with a Mn $d^{4}$ configuration and a Fermi surface dominated by $d$-like holes. Going into the details of the bandstructure we note that the triplet state at the Fermi level is made mainly from Mn $d$ states with $x y, x^{2}-y^{2}$, and $z^{2}$ 


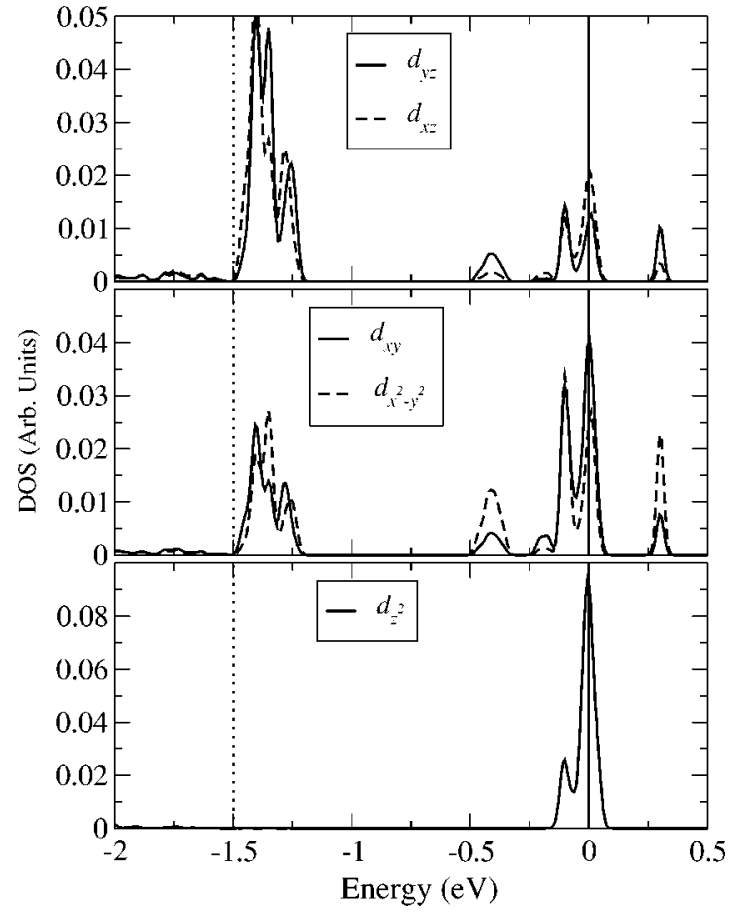

FIG. 6. Projected DOS for the Mn $d$ shell of a 3.125\% (Ga, Mn)N: LSDA results. The vertical lines denote, respectively, the position of the Fermi level (solid line) and of the GaN valence band top (dotted line).

symmetry, while the doublet is primarily made from $\mathrm{Mn} d_{x z}$ and $d_{y z}$ states. The $z$ axis has been taken here along the $c$ axis of the wurtzite structure. This can be easily seen from the density of states projected on the Mn $d$ shells (Figs. 6 and 7) and it is a direct consequence of the hexagonal crystal field splitting.

There are two main differences when going from LSDA to $\mathrm{LDA}+U$. First with $\mathrm{LDA}+U$ the $d_{x z}-d_{y z}$ doublet disappears at the top of the GaN valence band, leaving only the triplet in the band gap, which shifts closer to the valence band top. Secondly LDA $+U$ enhances the orbital ordering of the $x y, x^{2}-y^{2}, z^{2}$ triplet. In fact, while with LSDA this is a mixture of all the three orbital components, with the Fermi level cutting through a peak with strong $d_{z^{2}}$ character, in LDA $+U$ the Fermi level lies below the $d_{z^{2}}$ peak leaving a strongly localized $d_{z^{2}}$ hole. Secondly in LDA $+U$ there is a general reduction of the Mn $d$ component of the DOS at the Fermi level, with an increase of the $\mathrm{N} p$ component. In fact by integrating the orbital resolved DOS over the $x y, x^{2}-y^{2}, z^{2}$ triplet, we calculate that the relative contribution to the DOS coming from the Mn $d$ shells is 55 and $36 \%$, respectively, for LSDA and LDA $+U$.

These features suggest that the main effects of the Hubbard $U$ corrections are an increase of the localization of the Mn $d$ electrons associated with a reduction of both the onsite $d$ hybridization and the $\mathrm{Mn} d-\mathrm{N} p$ coupling. A similar behavior has also been found with the LDA-SIC method. ${ }^{20}$ However, in the LDA-SIC case the orbital ordering is much stronger producing a complete split of the triplet into an occupied doublet and an empty singlet (with mainly $d_{z^{2}}$ character). We have tried several values of $U$ and $J$ in order to

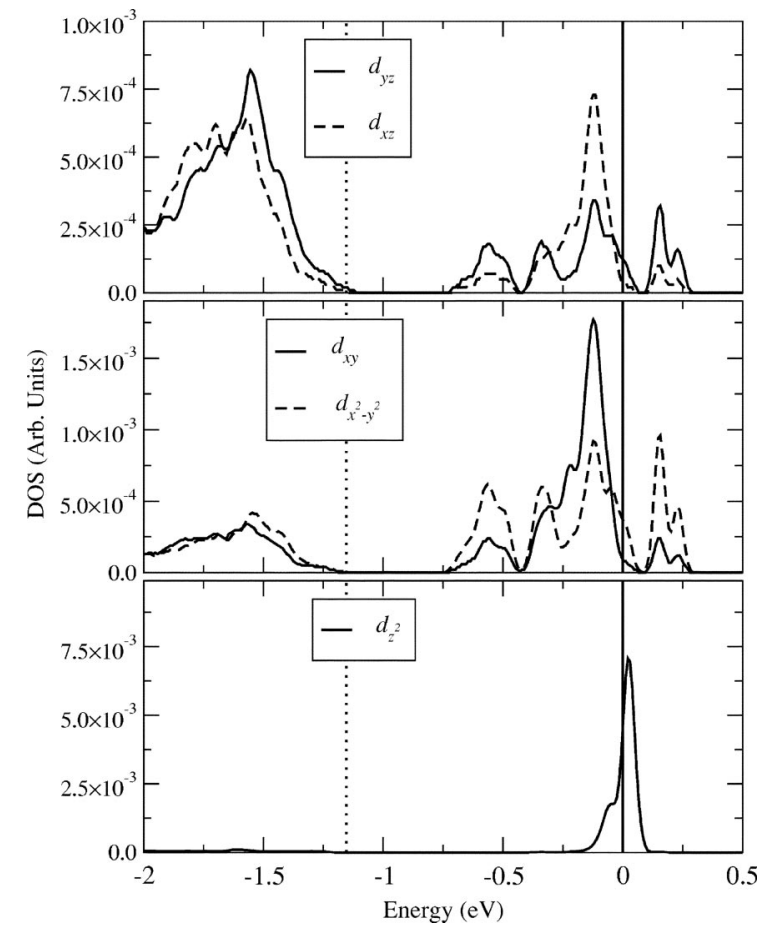

FIG. 7. Projected DOS for the Mn $d$ shell of a $3.125 \%$ (Ga,Mn) $\mathrm{N}$ : LDA $+U(U=4.5 \mathrm{eV}, J=1.0 \mathrm{eV})$ results. The vertical lines denote respectively the position of the Fermi level (solid line) and of the $\mathrm{GaN}$ valence band top (dotted line).

reproduce the LDA-SIC result, without success. The main difference between the two calculations is that in the case of LDA-SIC all the orbitals, including those of $\mathrm{Ga}$ and $\mathrm{N}$, are corrected. The resulting band gap of $\mathrm{GaN}$ is almost twice as big as that obtained with the simple LSDA. This means that the LDA-SIC and LDA $+U$ calculations start from a host semiconductor with a rather different band gap giving rise to a more pronounced orbital ordering in the LDA-SIC case. In support to this hypothesis it is worth reporting that novel LDA-SIC calculations, ${ }^{53}$ performed by subtracting the selfinteraction only from the $\mathrm{Mn} d$ orbitals, give very similar results than our present $\mathrm{LDA}+U$.

Also for $(\mathrm{Ga}, \mathrm{Mn}) \mathrm{N}$ we investigate the presence of an induced magnetic moment at the $\mathrm{N}$ sites. In this case the situation is rather different from that of $(\mathrm{Ga}, \mathrm{Mn})$ As. LSDA calculations show very little magnetic moment at any $\mathrm{N}$ sites, including nearest $\mathrm{Mn}$ neighbors where the induced magnetic moment is only $0.03 \mu_{B}$ and parallel to that of the Mn ion. Also LDA $+U$ gives small induced magnetic moments $\left(0.098 \mu_{B}\right.$ for the first neighbor sites, then smaller than $0.008 \mu_{B}$ for any other $\mathrm{N}$ ), however those are antiparallel to that of the Mn ions. More interestingly the occupation of the four $\mathrm{N}$ ions in the $\mathrm{MnN}_{4}$ tetrahedron is rather sensitive to whether the $\mathrm{N}$ ion is placed along the wurtzite $c$ axis (top $\mathrm{N}$ ) or in the opposite plane (in-plane $\mathrm{N}$ ). The induced magnetic moment is given solely by the $p_{z}$ orbital for the $\mathrm{N}$ top ion, and only by the $p_{x}$ and $p_{y}$ orbitals for the in-plane N. However, in contrast with the previous LDA-SIC results, ${ }^{20}$ in the present case the induced $\mathrm{N}$ magnetic moment is always antiparallel to that of the Mn. 


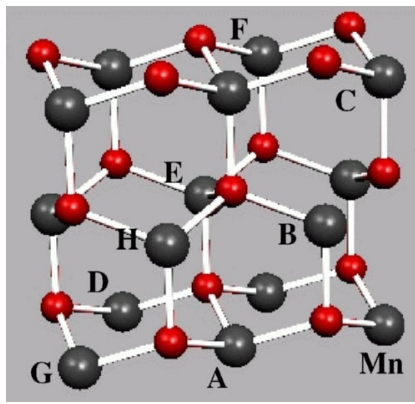

FIG. 8. Position of the Mn ions in the (Ga,Mn)N supercell. The large (small) spheres represent the possible Mn (N) sites. The first $\mathrm{Mn}$ ion is in the position "Mn" and the second occupies one of the sites labeled with a capital letter.

\section{D. (Ga,Mn)N: Magnetic interaction}

From the previous analysis it is clear that the band structure does not support a conventional Zener-like picture of the ferromagnetism, since no free holes are present in the GaN valence band. Therefore a careful investigation of the ferromagnetic coupling between $\mathrm{Mn}$ ions is needed. Also in this case we consider supercells where we include two Mn ions at different mutual positions, and we investigate the energy difference between the ferromagnetic and antiferromagnetic alignment of the Mn ions.

We explore several possible geometrical configurations, by using both a 32 and a 64 atom unit cell. The first Mn ion is always placed in the corner of the supercell and we allow the second to occupy different positions. Those possible con-

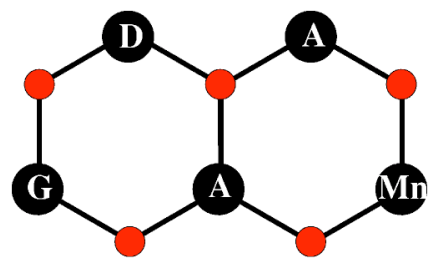

FIG. 9. Position of the $\mathrm{Mn}$ ions in the $(\mathrm{Ga}, \mathrm{Mn}) \mathrm{N}$ supercell: planar view. The large (small) circles represent the possible Mn (N) sites. The first $\mathrm{Mn}$ ion is in the position "Mn" and the second occupies one of the sites labeled with a capital letter.

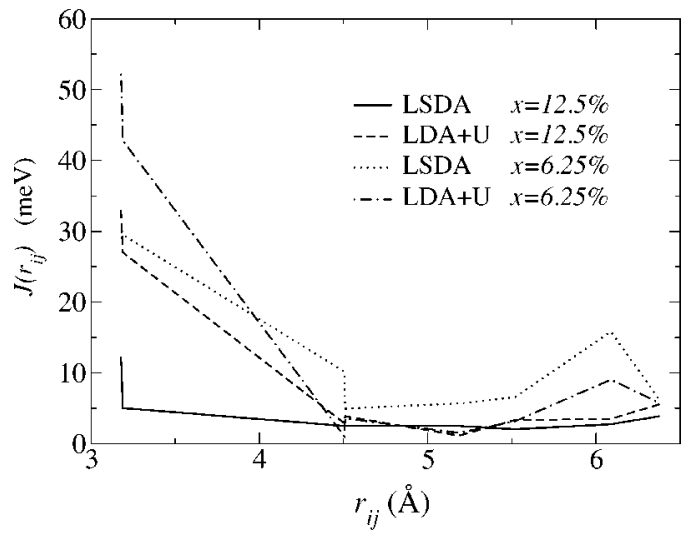

FIG. 10. Exchange constants $J$ as a function of the Mn-Mn separation for $x=12.5 \%$ and $x=6.25 \%$, obtained with either LSDA or LDA $+U$. The label indicated the position of the second $\mathrm{Mn}$ in the cell, according to Figs. 8 and 9, and Table I. The horizontal line denotes $J=0$.

figurations are schematically presented in Figs. 8 and 9. Also in this case we have fit our DFT energy calculations to the Heisenberg Hamiltonian of Eq. (3). In this case we use $\langle\vec{S}\rangle$ $=2$ and we have set $J(r)=0$ for $r>6.4 \AA$. A summary of the results for all the Mn positions studied here is presented in Table I and in Fig. 10.

From our data it is clear that the LDA $+U$ gives a ferromagnetic coupling with rather different features than that given by the LSDA. In particular when going from LSDA to $\mathrm{LDA}+U$ there is a large enhancement of the $J$ for nearest Mn neighbors (cases A and B), while all the other $J$ 's are either unchanged or reduced. A second interesting aspect is the dependence of $J(r)$ over the $\mathrm{Mn}$ concentration. Also in this case LSDA and LDA $+U$ give a rather different behavior. In LSDA all the exchange constants increase as the Mn concentration becomes smaller, while the LDA $+U$ gives an increase of the nearest neighbor constants and almost no dependence on concentration for all the other constants.

This strong dependence of the $J$ 's on the Mn-Mn distance, with strong coupling at short $\mathrm{Mn}-\mathrm{Mn}$ separation and weak long-range tails, suggests that high $T_{c}$ can be achieved only at reasonably large Mn concentrations, when it is more likely

TABLE I. Summary of the results for all the different configurations studied. The Mn positions correspond to those of Figs. 8 and 9 and $d$ is the Mn-Mn distance. In brackets we report the number of planes separating the two Mn ions along the wurtzite $c$ axis. All the values of $J$ are in meV, and the indexes label the Mn concentration $x$.

\begin{tabular}{llrrrr}
\hline \hline Position & $d(\AA)$ & $J_{0.125}^{\mathrm{LSDA}}$ & $J_{0.125}^{\mathrm{LDA}+U}$ & $J_{0.0625}^{\mathrm{LSDA}}$ & $J_{0.0625}^{\mathrm{LDA}+U}$ \\
\hline A (0) & 3.189 & 5.03 & 27.00 & 29.50 & 42.80 \\
B (1) & 3.179 & 12.30 & 32.99 & 30.00 & 52.23 \\
C (2) & 5.185 & 2.50 & 1.14 & 5.66 & 1.52 \\
D (0) & 4.510 & 2.48 & 3.87 & 4.98 & 3.56 \\
E (1) & 4.504 & 2.50 & 2.90 & 10.18 & 1.08 \\
F (2) & 6.088 & 2.75 & 3.50 & 15.83 & 9.05 \\
G (0) & 6.378 & 3.88 & 5.57 & 6.15 & 5.73 \\
H (1) & 5.518 & 2.04 & 3.33 & 6.55 & 3.16 \\
\hline \hline
\end{tabular}




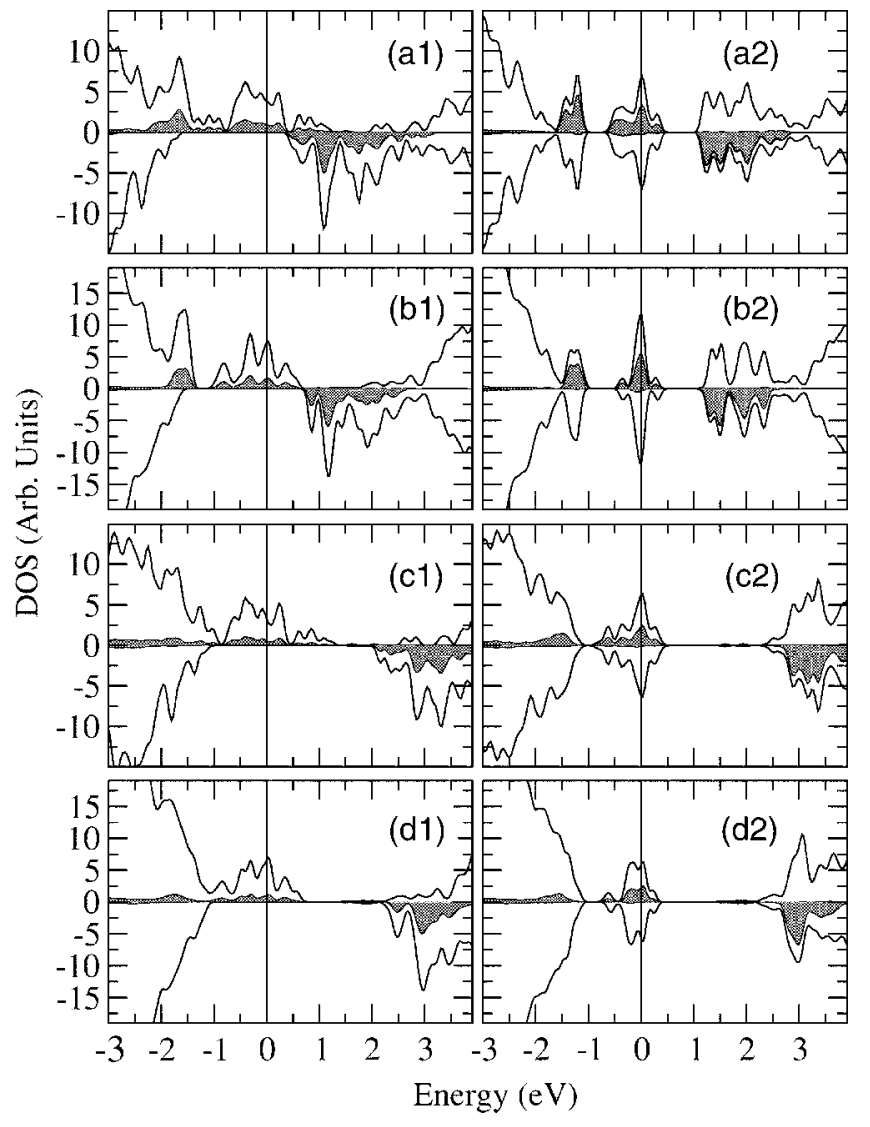

FIG. 11. Density of states for various 64 and 32 atom supercells containing two Mn ions in the position A (see Figs. 8 and 9). The different figures correspond to different $\mathrm{Mn}$ concentrations, to either LSDA or LDA $+U$ and to either parallel or antiparallel alignment of the Mn ions: (a1) $x=12.5 \%$ LSDA parallel, (a2) $x=12.5 \%$ LSDA antiparallel, (b1) $x=6.25 \%$ LSDA parallel, (b2) $x=6.25 \%$ LSDA antiparallel, (c1) $x=12.5 \% \mathrm{LDA}+U$ parallel, (c2) $x=12.5 \%$ LDA $+U$ antiparallel, (d1) $x=6.25 \%$ LDA $+U$ parallel, (d2) $x=6.25 \%$ LDA $+U$ antiparallel. The solid lines show the total DOS and the shadowed regions the contribution from the $d$ orbitals of one Mn ion. The horizontal lines denote the position of the Fermi level $(0 \mathrm{eV})$.

to have several Mn ions occupying nearest-neighboring positions. This leads us to speculate that the ferromagnetic state occurs in ferromagnetic clusters (with wurtzite lattice structure), presenting very large $\mathrm{Mn}$ concentration. Within the clusters the ferromagnetic state is stabilized by the strong nearest-neighbor ferromagnetic interaction, while the clustercluster coupling remains weak. Therefore our LDA $+U$ calculations support the hypothesis of two magnetic phases in $(\mathrm{Ga}, \mathrm{Mn}) \mathrm{N}:{ }^{12}$ a high $T_{c}$ ferromagnetic phase characterized by large Mn concentrations, and a low $T_{c}$ ferromagnetic or even paramagnetic phase obtained at much lower concentrations.

To gain more insights into the nature of the ferromagnetic coupling we have studied in great details the electronic structure of various supercells containing two Mn ions. Here we focus only on the case of nearest neighbor Mn ions, which are the ones presenting the larger exchange constants, and in particular on the case A. In Fig. 11 we present the DOS for both 64 and 32 atom cells containing two Mn ions in the position A. The picture shows the results obtained with LSDA (top four panels) and LDA $+U$ (bottom four panels) for both the parallel (right panels) and antiparallel (left panels) alignments of the Mn spins.

From the picture it is clear that the ferromagnetic state becomes more stable when the ferromagnetic configuration presents a large spin gap, i.e., when there is little overlap between the two Mn derived spin subbands. This is the case of LDA $+U$ for both the concentrations studied, where we find a spin gap of the order of $\sim 1.5 \mathrm{eV}$. Moreover in $\mathrm{LDA}+U$ this spin gap appears to be rather insensitive to the Mn concentration (although it clearly increases when going from $x=12.5$ to $6.25 \%$ ), which reflects the corresponding weak dependence of $J$.

In contrast in the LSDA case there is a substantial overlap between the majority and the minority Mn $d$ bands, in particular at large Mn concentrations. This is a direct consequence of the broadening of the Mn $d$ states, upon increasing concentration. It is worth noting that in our 32 atom cell each $\mathrm{Mn}$ ion has two Mn neighbors separated only by one $\mathrm{N}$ site forming a Mn-N chain, whereas in the 64 atom cell Mn$\mathrm{N}-\mathrm{Mn}$ trimers are separated by two $\mathrm{N}$ sites. The large broadening obtained for $x=12.5 \%$ closes almost entirely the spin gap, resulting in a rather weak ferromagnetic coupling between the Mn.

These findings can be understood in terms of competition between the superexchange and double-exchange mechanisms. ${ }^{54,55}$ In absence of any holes the coupling between two $\mathrm{Mn}$ ions is expected to be antiferromagnetic due to the superexchange coupling, as was recently verified. ${ }^{22}$ This coupling is extremely short ranged and one has to assume that $\mathrm{Mn}$ ions separated by more than one $\mathrm{N}$ site are magnetically decoupled. Doping such a system will generally produce a distortion of the antiferromagnetic coupling, eventually leading to a ferromagnetic ground state for large enough dopants concentration. The "melting" of the antiferromagnetic state is connected with the fact that the additional electrons (or holes) are exchange coupled with the local spin of the transition metal impurities. In this case the wave function of such electrons depends on the magnetic configuration of the transition metal ions, and in particular it will be localized when those are aligned along different directions. The formation of a ferromagnetic state will enhance the delocalization of the additional electrons providing a net gain in band energy. The final ground state is then the result of the competition between the energy gain due to the electron delocalization and the energy loss due to direct Mn-Mn superexchange. Therefore in $(\mathrm{Ga}, \mathrm{Mn}) \mathrm{N}$ the ferromagnetic ground state is directly connected with the presence of a hole in the Mn-derived impurity band.

However, in the case of small spin splitting of the Mn $d$ states, virtual hopping of the holes between antiferromagnetically oriented $\mathrm{Mn}$ ions becomes possible and their kinetic energy can be lowered without producing a ferromagnetic ground state. This is why the LSDA calculations for large Mn doping present a rather small ferromagnetic interaction between the Mn ions. In addition this enhanced hopping between antiferromagnetically aligned $\mathrm{Mn}$ ions produces a reduction of the Mn magnetic moment. For the $x=12.5 \%$ case LSDA shows a magnetic moment of the Mn $d$ shell (obtained 
from the Mülliken population) of $3.9 \mu_{B}$ and $3.2 \mu_{B}$, respectively, for the ferromagnetic and antiferromagnetic configuration. This is indeed very different from the $\mathrm{LDA}+U$ case, where the local magnetic moment changes little with the mutual Mn alignment.

We have performed the same analysis over all the configurations studied with very similar conclusions. In the other case of short Mn-Mn distance (case B) the tiny differences with the case A are due to the different effective Mn-Mn hopping integral. This is expected since the hopping integral depends on the specific orbitals forming the bond and ultimately on the path connecting the two Mn ions. This gives rise to a weak anisotropy in the magnetic coupling. Finally for $\mathrm{Mn}$ ions separated by more than one $\mathrm{N}$ site the coupling is always rather small due to the small hopping integral.

\section{SUMMARY}

We have investigated extensively the mechanism for the ferromagnetic coupling in $(\mathrm{Ga}, \mathrm{Mn}) \mathrm{As}$ and $(\mathrm{Ga}, \mathrm{Mn}) \mathrm{N}$, by using density functional theory in both the standard LSDA and our newly implemented LDA $+U$ method.

For $(\mathrm{Ga}, \mathrm{Mn}) \mathrm{As} \mathrm{LDA}+U$ qualitatively does not change the general picture given by LSDA. Both methods confirm a strong $p$ - $d$ hybridization leading to a spin splitting of the valence band of GaAs. In this case a hole mediated Zener model for the ferromagnetism is appropriate. After having fixed the Coulomb and exchange constants $U$ and $J$ to values that reproduce accurately both the position of the Mn $d$ shell coming from photoemission data ${ }^{41}$ and the spin splitting of the GaAs valence band coming from the LDA-SIC method, ${ }^{20}$ we have estimated the Zener mean-field exchange parameter $N \beta$ to be $\sim-2.8 \mathrm{eV}$. We believe this is the correct value that should be used in model Hamiltonian calculations.

Then we moved our attention to $(\mathrm{Ga}, \mathrm{Mn}) \mathrm{N}$. In this case the addition of the on-site $U$ corrections results in a very strong, short range, ferromagnetic coupling between the Mn ions. This is rather anisotropic and decays quickly with the $\mathrm{Mn}-\mathrm{Mn}$ separation. The strong ferromagnetic interaction is double-exchange-like and is associated with the creation of a wide Mn- $d / \mathrm{N}-p$ impurity band at the Fermi level. These features sustain a picture of the ferromagnetism where a high- $T_{c}$ ferromagnetic phase given by regions with large Mn concentration, coexist with a low $T_{c}$ ferromagnetic or perhaps paramagnetic phase given by small $\mathrm{Mn}$ concentration regions.

\section{ACKNOWLEDGMENTS}

This work is sponsored by Enterprise Ireland under the grant EI-SC/2002/10. Traveling is sponsored by Enterprise Ireland under the International Collaboration programme EIIC/2003/47. D.S.-P. acknowledges support by the Basque Departamento de Educación, the UPV/EHU (Grant. No. 9/UPV 00206.215-13639/2001), and the Spanish MEC (Grant No. FIS2004-06490-C03-02).

\section{APPENDIX A: LDA $+\boldsymbol{U}$ IMPLEMENTATION IN THE SIESTA CODE}

The LDA $+U$ method combines LSDA density functional theory with the impurity Anderson model. The main idea is to divide the electronic states into two subsystems: localized (generally $d$ or $f$ ) and delocalized electrons (generally $s$ and $p$ ). In what follows we always refer to the localized orbitals as $d$ orbitals. Then the LDA $+U$ philosophy consists in replacing the averaged (LSDA) Coulomb and exchange interactions acting on the localized shells, by an orbital dependent Hartree-Fock-like Hamiltonian. ${ }^{39,40}$ The generalized LDA $+U$ functional is defined as

$$
E^{\mathrm{LDA}+U}\left[\rho^{\sigma}(\vec{r}),\left\{n^{\sigma}\right\}\right]=E^{\mathrm{LSDA}}\left[\rho^{\sigma}(\vec{r})\right]+E^{U}\left[\left\{n^{\sigma}\right\}\right]-E^{\mathrm{DC}}\left[\left\{n^{\sigma}\right\}\right],
$$

where $E^{\mathrm{LSDA}}$ is the standard LSDA functional, $\rho^{\sigma}(\vec{r})$ is the charge density for the spin $\sigma$ electrons, and $\left\{n^{\sigma}\right\}$ is the reduced density matrix corresponding to the orbitals we need to correct. Finally $E^{U}$ and $E^{\mathrm{DC}}$ are respectively the new Hubbard-like functional and the double counting correction. $E^{\mathrm{DC}}$ is necessary to eliminate the averaged electron-electron interaction within the $d$ shell, which is already included in $E_{\text {LSDA }}$. Following Anisimov et al. the total energy of a spin polarized system can be written as

$$
\begin{aligned}
E_{\mathrm{LDA}+\mathrm{U}}= & E_{\mathrm{LSDA}}+\frac{1}{2} \sum_{m m^{\prime} \sigma} U_{m m^{\prime}} n_{m \sigma} n_{m^{\prime}-\sigma} \\
& +\frac{1}{2} \sum_{m \neq m^{\prime} \sigma}\left(U_{m m^{\prime}}-J_{m m^{\prime}}\right) n_{m \sigma^{\prime}} n_{m^{\prime} \sigma} \\
& -U\left[N^{\uparrow}\left(N^{\uparrow}-1\right) / 2+N^{\downarrow}\left(N^{\downarrow}-1\right) / 2+N^{\uparrow} N^{\downarrow}\right] \\
& +J\left[N^{\uparrow}\left(N^{\uparrow}-1\right) / 2+N^{\downarrow}\left(N^{\downarrow}-1\right) / 2\right],
\end{aligned}
$$

where $N^{\uparrow}$ and $N^{\downarrow}$ are total number of, respectively, spin-up and spin-down electrons occupying the $d$ shell, and $n_{m \sigma}$ and $n_{m-\sigma}$ are the orbital occupation numbers, which are calculated self-consistently within the LDA $+U$ approach. It is assumed in the spirit of the LDA $+U$ approximation that the total occupations of the $d$ shell $N^{\sigma}$ are identical within LSDA and LDA $+U$. This assumption justifies the definition of the double counting term $E^{\mathrm{DC}}$.

The index $m$ runs over the magnetic quantum number. The parameters $U$ and $J$ are, respectively, the Coulomb and the exchange interaction constants, that in principle can be calculated as the two-electron matrix elements of the atomic electron-electron interaction potential $V_{e e}$

$$
\begin{aligned}
& U_{m m^{\prime}}=\left\langle m m^{\prime}\left|V_{e e}\right| m m^{\prime}\right\rangle, \\
& J_{m m^{\prime}}=\left\langle m^{\prime} m\left|V_{e e}\right| m m^{\prime}\right\rangle .
\end{aligned}
$$

The effective $\mathrm{LDA}+U$ potential in then obtained by taking the functional derivative of the total energy $E_{\mathrm{LDA}+U}$ with respect to the orbital density $n_{m \sigma}(\mathbf{r})$. This yields 


$$
\begin{aligned}
V_{m \sigma}= & V_{\mathrm{LSDA}}-U\left(N-\frac{1}{2}\right)+J\left(N^{\sigma}-\frac{1}{2}\right) \\
& +\sum_{m^{\prime} \neq m}\left(U_{m m^{\prime}}-J_{m m^{\prime}}\right) n_{m^{\prime} \sigma}+\sum_{m^{\prime}} U_{m m^{\prime}} n_{m^{\prime}-\sigma} .
\end{aligned}
$$

In our implementation we assume $U$ and $J$ to be independent from the magnetic quantum number $m$, although of course they can be different for $d$ and $f$ shells. Hence if $U_{m m^{\prime}}=U$ and $J_{m m^{\prime}}=J$, the equation above becomes

$$
\begin{aligned}
V_{m \sigma}= & V_{\mathrm{LSDA}}+U \sum_{m^{\prime}}\left(n_{m^{\prime}-\sigma}-n_{0-\sigma}\right) \\
& +(U-J) \sum_{m^{\prime} \neq m}\left(n_{m^{\prime} \sigma}-n_{0 \sigma}\right)+(U-J)\left(\frac{1}{2}-n_{0 \sigma}\right)
\end{aligned}
$$

with $n_{0 \sigma}$ the average orbital occupations of the correlated shell

$$
n_{0 \sigma}=\frac{1}{2 l+1} N^{\sigma},
$$

and $l$ the orbital quantum number.

From the potential of Eq. (A6) one can extract an intuitive picture of the effects of strong correlations on the oneparticle energy levels. We have

$$
\epsilon_{m \sigma}^{\mathrm{LDA}+U}=\epsilon_{m \sigma}^{\mathrm{LSDA}}+(U-J)\left(\frac{1}{2}-n_{m \sigma}\right),
$$

where $n_{m \sigma}$ are the LDA $+U$ orbital occupations. In the simple formula above the single particle energies of the occupied and unoccupied orbitals are shifted, respectively, by
$-1 / 2(U-J)$ and $+1 / 2(U-J)$ reproducing qualitatively the correct physics of a Mott-Hubbard insulator.

We turn now our attention to the numerical implementation of this method in SIESTA. This is quite straightforward since SIESTA uses a localized atomic orbital basis set. ${ }^{27,29}$ Let us call these nonorthogonal basis functions $\{\chi\}$. The twocenter overlap integrals $S_{\mu \nu}$ are then given by

$$
S_{\mu \nu}=\int \chi_{\mu}\left(\mathbf{r}-\mathbf{R}_{1}\right) \chi_{\nu}\left(\mathbf{r}-\mathbf{R}_{2}\right) d \mathbf{r},
$$

where $\mathbf{R}_{1}$ and $\mathbf{R}_{2}$ are the atomic centers and the density matrix in our atomic functions representation is denoted as $D_{\mu \nu}$. The occupation number of a given atomic orbital $m$ is then defined as

$$
n_{m \sigma}=\sum_{\mu \nu} S_{m \mu} D_{\mu \nu}^{\sigma} S_{\nu m} .
$$

The additional potential of Eq. (A6) is an operator $\hat{V}_{m \sigma}$ of the form

$$
\hat{V}_{m \sigma}=V_{m \sigma}|m \sigma\rangle\langle m \sigma|,
$$

where $V_{m \sigma}$ is the scalar defined in Eq. (A6) and $|m \sigma\rangle\langle m \sigma|$ is the projector on the molecular state $m$ with spin $\sigma$. Assuming $|m \sigma\rangle$ to be one of our basis functions the matrix element of the $\mathrm{LDA}+U$ potential can be written as

$$
\left(\hat{V}_{m \sigma}\right)_{\mu \nu}=S_{\mu m} V_{m \sigma} S_{m \nu}
$$

In this case we use a multiple- $\zeta$ basis set for the localized shell, and we construct the LDA $+U$ projector from one of the $\zeta$. The cutoff radius of this particular basis function is usually much shorter than that of the other basis functions, and in this work we have used projectors with a cutoff radius of 2.2 Bohr.
*Present address: INFM DEMOCRITOS National Simulation Center, via Beirut 2-4, 34014 Trieste, Italy.

†Electronic address: sanvitos@tcd.ie

${ }^{1}$ T. Dietl, Semicond. Sci. Technol. 17, 377 (2002).

${ }^{2}$ H. Ohno, Science 281, 951 (1998).

${ }^{3}$ W. Prellier, A. Fouchet, and B. Mercey, J. Phys.: Condens. Matter 15, R1583 (2003).

${ }^{4}$ H. Ohno, D. Chiba, F. Matsukura, T. Omiya, E. Abe, T. Dietl, Y. Ohno, and K. Ohtani, Nature (London) 408, 944 (2000).

${ }^{5}$ K. Ando, T. Hayashi, M. Tanaka, and A. Twardowski, J. Appl. Phys. 83, 6548 (1998).

${ }^{6}$ M. L. Reed, N. A. El-Masry, H. H. Stadelmaier, M. K. Ritums, M. J. Reed, C. A. Parker, J. C. Roberts, and S. M. Bedair, Appl. Phys. Lett. 79, 3473 (2001).

${ }^{7}$ G. T. Thaler, M. E. Overberg, B. Gila, R. Frazier, C. R. Abernathy, S. J. Pearton, J. S. Lee, S. Y. Lee, Y. D. Park, Z. G. Khim, J. Kim, and F. Ren, Appl. Phys. Lett. 80, 3964 (2002).

${ }^{8}$ S. Sonoda, S. Shimizu, T. Sasaki, Y. Yamamoto, and H. J. Hori, J. Cryst. Growth 237-239, 1358 (2002).

${ }^{9}$ S. Sonoda, H. Hori, Y. Yamamoto, T. Sasaki, M. Sato, S. Shimizu,
K. Suga, and K. Kindo, IEEE Trans. Magn. 38, 2859 (2002).

${ }^{10}$ T. Dietl, H. Ohno, F. Matsukura, J. Cibert, and D. Ferrand, Science 287, 1019 (2000).

${ }^{11}$ C. Zener, Phys. Rev. 81, 440 (1950); 83, 299 (1950).

${ }^{12}$ K. Ando, Appl. Phys. Lett. 82, 100 (2003).

${ }^{13}$ J. König, H.-H. Lin, and A. H. MacDonald, Phys. Rev. Lett. 84, 5628 (2000).

${ }^{14}$ S. Sanvito, G. Theurich, and N. A. Hill, J. Supercond Novel Magn. Mater. 15, 85 (2002).

${ }^{15}$ S. Sanvito, P. Ordejón, and N. A. Hill, Phys. Rev. B 63, 165206 (2001).

${ }^{16}$ M. van Schilfgaarde and O. N. Myrasov, Phys. Rev. B 63, 233205 (2001).

${ }^{17}$ M. Jain, L. Kronik, J. R. Chelikowsky, and V. V. Godlevsky, Phys. Rev. B 64, 245205 (2001).

${ }^{18}$ K. Sato, H. Katayama-Yoshida, and P. H. Dederichs, J. Supercond Novel Magn. Mater. 16, 31 (2003).

${ }^{19}$ L. Kronik, M. Jain, and J. R. Chelikowsky, Phys. Rev. B 66, 041203(R) (2002). 
${ }^{20}$ A. Filippetti, N. A. Spaldin, and S. Sanvito, cond-mat/0302178 (unpublished).

${ }^{21}$ A. Filippetti and N. A. Spaldin, Phys. Rev. B 67, 125109 (2003).

${ }^{22}$ L. M. Sandratskii, P. Bruno, and K. Kudrnovsky, Phys. Rev. B 69, 195203 (2004).

${ }^{23}$ B. Sanyal, O. Bengone, and S. Mirbt, Phys. Rev. B 68, 205210 (2003).

${ }^{24}$ P. Mahadevan and A. Zunger, Phys. Rev. B 69, 115211 (2004).

${ }^{25}$ K. Sato, P. H. Dederichs, H. Katayama-Yoshida, and J. Kudrnovsky, Physica B 340-342, 863 (2003).

${ }^{26}$ D. Sánchez-Portal, P. Ordejón, E. Artacho, and J. M. Soler, Int. J. Quantum Chem. 65, 453 (1997).

${ }^{27}$ J. M. Soler, E. Artacho, J. D. Gale, A. García, J. Junquera, P. Ordejón, and D. Sánchez-Portal, J. Phys.: Condens. Matter 14, 2745 (2002).

${ }^{28}$ O. F. Sankey and D. J. Niklewski, Phys. Rev. B 40, 3979 (1989).

${ }^{29}$ J. Junquera, O. Paz,D. Sánchez-Portal, and E. Artacho, Phys. Rev. B 64, 235111 (2001).

${ }^{30}$ E. Anglada, J. M. Soler, J. Junquera, and E. Artacho, Phys. Rev. B 66, 205101 (2002).

${ }^{31}$ L. Kleinman and D. M. Bylander, Phys. Rev. Lett. 48, 1425 (1982).

${ }^{32}$ D. Sánchez-Portal, R. M. Martin, S. M. Kauzlarich, and W. E. Pickett, Phys. Rev. B 65, 144414 (2002).

${ }^{33}$ N. Troullier and J. L. Martins, Phys. Rev. B 43, 1993 (1991).

${ }^{34}$ S. G. Louie, S. Froyen, and M. L. Cohen, Phys. Rev. B 26, 1738 (1982).

${ }^{35}$ A. García and M. L. Cohen, Phys. Rev. B 47, 6751 (1993)

${ }^{36}$ W. R. L. Lambrecht, B. Segal, S. Strite, G. Martin, A. Agarwal, H. Morkoc, and A. Rockett, Phys. Rev. B 50, 14155 (1994).

${ }^{37}$ S. J. Pearton, C. R. Abernathy, M. E. Overberg, G. T. Thaler, D. P. Norton, N. Theodoropou, A. F. Hebard, Y. D. Park, F. Ren, J. Kim, and L. A. Boatner, J. Appl. Phys. 93, 1 (2002).
${ }^{38}$ E. Artacho, D. Sánchez-Portal, P. Ordejón, A. García, and J. M. Soler, Phys. Status Solidi B 215, 809 (1999).

${ }^{39}$ V. I Anisimov, J. Zaanen, and O. K. Andersen, Phys. Rev. B 44, 943 (1991).

${ }^{40}$ V. I. Anisimov, F. Aryasetiawan, and A. I. Lichtenstein, J. Phys.: Condens. Matter 9, 767 (1997).

${ }^{41}$ J. Okabayashi, A. Kimura, O. Rader, T. Mizokawa, A. Fujimori, T. Hayashi, and M. Tanaka, Phys. Rev. B 64, 125304 (2001).

${ }^{42}$ S. Das Sarma, E. H. Hwang, and A. Kaminski, Phys. Rev. B 67, 155201 (2003).

${ }^{43}$ T. Dietl, F. Matsukura, and H. Ohno, Phys. Rev. B 66, 033203 (2002).

${ }^{44}$ R. S. Mülliken, J. Chem. Phys. 23, 1833 (1955); 23, 1841 (1955).

${ }^{45}$ V. Bernoit á la Guillaume, D. Scalbert, and T. Dietl, Phys. Rev. B 46, 9853 (1992).

${ }^{46}$ T. Omiya, F. Matsukura, T. Dietl, Y. Ohno, T. Sakon, M. Motokawa, and H. Ohno, Physica E (Amsterdam) 7, 976 (2000).

${ }^{47}$ F. Matsukura, H. Ohno, A. Shen, and Y. Sugawara, Phys. Rev. B 57, R2037 (1998).

${ }^{48}$ J. Szczytko, W. Mac, A. Stachow, A. Twardowski, P. Becla, and J. Tworzydło, Solid State Commun. 99, 927 (1996).

${ }^{49}$ J. Okabayashi, A. Kimura, O. Rader, T. Mizokawa, A. Fujimori, T. Hayashi, and M. Tanaka, Phys. Rev. B 58, R4211 (1998).

${ }^{50}$ S. Sanvito and N. A. Hill, Appl. Phys. Lett. 78, 3493 (2001).

${ }^{51}$ L. M. Sandratskii and P. Bruno, Phys. Rev. B 67, 214402 (2003).

${ }^{52}$ S. Sanvito, Phys. Rev. B 68, 054425 (2003).

${ }^{53}$ Alessio Filippetti (private communication).

${ }^{54}$ M. T. Czyżyk and G. A. Sawatzky, Phys. Rev. B 49, 14211 (1994).

${ }^{55}$ D. I Khomski and G. A. Sawatzky, Solid State Commun. 102, 87 (1997). 